\title{
Sequencing with graphene pores
}

\author{
Solid-state nanopores are often used for biomolecular analysis, but have so far been unable to sequence DNA. \\ Marija Drndić asks whether nanopores made in graphene could fulfil all of the requirements needed for sequencing.
}

$\mathrm{S}$ olid-state nanopores can be used to detect, analyse and manipulate nanoscale objects, and could be used in applications such as nanoparticle analysis and water filtration. They are also of particular interest in the sequencing of DNA. In a typical experiment, a DNA molecule is threaded through a nanoscale hole in a membrane under an applied voltage, and the ion current flowing through this pore is monitored; when the DNA nucleotides enter the pore they block the current by different amounts, and this signal could then be used to identify the individual bases and in turn the DNA sequence.

The field of solid-state nanopores emerged from work on Coulter counters ${ }^{1}$, ion channels and protein pores ${ }^{2-5}$, and recently proof-ofprinciple DNA sequencing measurements have been reported using ionic currents and protein pores ${ }^{6-8}$. Compared with protein nanopores, solid-state nanopores offer potential advantages such as robustness, size tunability and compatibility with large-scale chip manufacturing. But solid-state nanopores cannot yet be used to identify individual bases. This is due, in part, to the fact that the membranes used are too thick to probe the local structure of the DNA; they are typically silicon nitride membranes with thicknesses of more than $10 \mathrm{~nm}$, which is equivalent to about 30 DNA bases. As a result, researchers have turned to thin membranes such as graphene, which, because of its atomic thickness, could potentially yield distinct current signals from one or a few DNA bases.

In 2010, three independent research groups - led by Jene Golovchenko, Cees Dekker and myself - reported threading double-stranded DNA molecules through nanopores in suspended graphene membranes $^{9-11}$ (Fig. 1). Although there were differences in the experiments - such as whether the graphene was grown ${ }^{9,11}$ or exfoliated $^{12}$, and the diameters of the nanopores used - the underlying conclusions were similar and showed that the ionic currents could be used to detect the DNA.

In all three of these experiments, the DNA passed through the nanopore too quickly for individual bases to be resolved, and achieving single-base resolution with graphene nanopores remains an unresolved challenge. The percentage of graphene nanopores allowing DNA translocation is also lower than

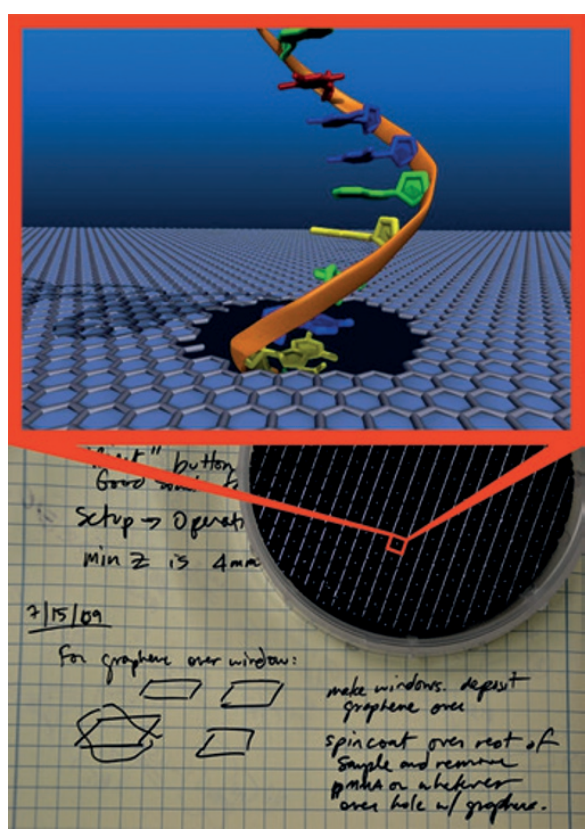

and signal-to-noise ratios increased at high bandwidths ${ }^{19}$, or the translocation of the DNA needs to be slowed down without more noise being introduced into the signal. There are also more prosaic but crucial details that need to be dealt with: enough devices have to be tested and reproduced by independent labs to draw firm conclusions, a task that requires skill, time and effort.

At this stage, it is not clear what solidstate nanopore material will be able to meet all of these challenges or, in fact, whether new nanopores will have to be developed to achieve them. There is, however, no theorem stating that only one material will work. More likely, if one works out, others may too - although we should keep in mind that, while it is encouraging that protein pores have shown proof-of-principle sequencing, this is not a proof that any of the solid-state pores will work. And although efforts were undertaken to commercialize nanopores even before any proof-of-principle sequencing demonstration existed, and it is reasonable to extrapolate from existing data, only reproducible experiments can prove a hypothesis.

Danopore Bott wafer with hundreds of nanopore chips, and laboratory notebook entry ${ }^{9}$.

in typical silicon nitride nanopores, mostly owing to wetting problems ${ }^{9}$. Furthermore, more work is needed before graphene nanopores will match the latest achievements of traditional solid-state nanopores made from silicon nitride ${ }^{12-14}$ in terms of signal level and signal-to-noise ratio. Nevertheless, the work on graphene nanopores has already inspired a search for other thin membrane materials, and nanopores have, for example, been created from hafnium oxide ${ }^{15,16}$, molybdenum disulphide ${ }^{17}$ and boron nitride ${ }^{18}$ membranes.

For any solid-state nanopores - be it graphene, silicon nitride or something else - there is still a variety of fundamental issues that need to be resolved before DNA sequencing with ionic currents could become a reality. For example, should single- or double-stranded DNA be used? Is a specific range of nanopore diameters required, and how precisely will they have to be manufactured? Improvements in the nanopore devices will also be required. In particular, current noise needs to be lowered
Marija Drndic is in the Department of Physics and Astronomy, David Rittenhouse Laboratory, University of Pennsylvania, Philadelphia,

Pennsylvania 19104, USA.

e-mail:drndic@physics.upenn.edu

\section{References}

1. Coulter, W. H. Means for counting particles suspended in a fluid. US patent 2,656,508 (1953).

2. Kasianowicz, J. J., Brandin, E., Branton, D. \& Deamer, D. W. Proc. Natl Acad. Sci. USA 93, 13770-13773 (1996)

3. Akeson, M., Branton, D., Kasianowicz, J. J., Brandin, E. \& Deamer, D. W. Biophys. J. 77, 3227-3233 (1999).

4. Deamer, D. W. \& Akeson, M. Trends Biotechnol. 18, 147-151 (2000).

5. Deamer, D. W. \& Branton, D. Acc. Chem. Res. 35, 817-825 (2002)

6. Cherf, G. M. et al. Nature Biotechnol. 30, 344-348 (2012).

Manrao, E. A. et al. Nature Biotechnol. 30, 349-353 (2012).

8. Laszlo, A. H. et al. Nature Biotechnol. 32, 829-833 (2014).

9. Merchant, C. A. et al. Nano Lett. 10, 2915-2921 (2010).

10. Schneider, G. F. et al. Nano Lett. 10, 3163-3167 (2010).

11. Garaj, S. et al. Nature $467,190-193$ (2010).

12. Wanunu, M. et al. Nature Nanotech. 5, 807-814 (2010).

13. Venta, K. et al. ACS Nano 7, 4629-4636 (2013).

14. Carlsen, A. T., Zahid, O. K., Ruzicka, J., Taylor, E. W. \& Hall, A. R. ACS Nano 8, 4754-4760 (2014)

15. Shim, J., Rivera, J. A. \& Bashir, R. Nanoscale 5, 10887-10893 (2013)

16. Larkin, J. et al. ACS Nano 7, 10121-10128 (2013)

17. Liu, K., Feng, J., Kis, A. \& Radenovic, A. ACS Nano 8, 2504-2511 (2014)

18. Liu, S. et al. Adv. Mater. 25, 4549-4554 (2013).

19. Rosenstein, J. K., Wanunu, M., Merchant, C. A., Drndić, M. \& Shepard, K. L. Nature Methods 9, 487-492 (2012). 